ARTÍCULO ORIGINAL
REVISTACDLIMBIANA
DE CIENCIAANIMAL

Rev Colombiana Cienc Anim 2017; 9(1):66-72.

\title{
Casuística clínica más frecuente en el servicio ambulatorio de grandes animales de la Universidad de Córdoba, Colombia
}

\section{Clinical casuistic most frequently in the clinic large animal of the University of Córdoba, Colombia}

\author{
Cardona A, José ${ }^{*}$ Ph.D, Martínez M, Mastoby² M.Sc, Maza A, Libardo ${ }^{3}$ M.Sc.
}

Universidad de Córdoba, Facultad de Medicina Veterinaria y Zootecnia, Departamento de Ciencias Pecuarias, Montería, Colombia. ${ }^{1}$ Grupo de Investigación en Medicina de Grandes Animales (MEGA), ${ }^{2}$ Grupo de investigación MECIVET.

${ }^{3}$ Grupo de investigación en Producción Animal Tropical, Línea de Nutrición y Alimentación Animal "NUTRA".

\section{Key words:}

Cattle; equine; frequency; clinical cases.

\section{Palabras Clave:}

Bovinos; equinos; frecuencia; casos clínicos.

INFORMACIÓN Recibido: 04-02-2016; Aceptado: 08-07-2016. Correspondencia autor: cardonalvarez@hotmail.com

\begin{abstract}
The main objective was to identify the frequency of diseases commonly encountered, was conducted large animals from different farms in the department of Cordoba, Colombia. Between 2008 and 2015, were evaluated clinical, epidemiological and laboratorial, 2795 cattle and 513 horses in order to establish their respective diagnosis and appropriate management strategy. The data were classified by species and affected system, with cattle having more patients ( $84.5 \%)$, followed by horses $(15.5 \%)$. Therefore the most common pathological conditions found in these animals and their probable causes are described. Among them, the most important for both species were the Hematopoietic system where endoglobular hematropicos (Babesia sp. and Anaplasma sp.) were the main cause. $5 \%$ of the patients received surgical treatment, with the skin and exhibits the surgery, while $95 \%$ of cases received medical treatment. Cases seen in the medicalsurgical area between 2008 and 2015 was high, so tracking problems facing large animals in the Department of Córdoba is necessary and delivery of short courses is recommended training centers for small and medium producers, in order to improve management systems and prophylaxis of animal work and production.
\end{abstract}

\section{Resumen}

El objetivo principal fue identificar la frecuencia de las patologías comúnmente encontradas en los grandes animales de las diferentes explotaciones ganaderas del departamento de Córdoba, Colombia. Entre los años 2008 y 2015, fueron evaluados clínicamente, epidemiológicamente y con pruebas diagnósticas, 2795 bovinos y 513 equinos con el fin de establecer su respectivo diagnóstico y la estrategia de manejo adecuada. Los datos fueron clasificados por especie y sistema afectado, siendo los bovinos los de mayor casuística $(84,5 \%)$, seguido por los equinos (15,5\%). Razón por la cual se describen las condiciones patológicas más frecuentes encontradas en estos animales y sus causas probables. Los casos más destacados fueron para ambas especies en el sistema Hematopoyético, donde los hematropicos endoglobulares (Babesia spp y Anaplasma spp) fueron la principal causa. El $5 \%$ de los pacientes recibió tratamiento quirúrgico, siendo la piel y anexos los más intervenidos, mientras que el 95\% de los casos recibió tratamiento médico. La casuística atendida en el área de médico-quirúrgica entre los años 2008 y 2015 fue elevada, por lo que se hace necesario el seguimiento de los problemas que aquejan a los grandes animales en el Departamento de Córdoba y se recomienda la impartición de cursos cortos en centros de adiestramiento para los medianos y pequeños productores, con el fin de que mejoren los sistemas de manejo y profilaxis de los animales de trabajo y producción. 


\section{Introducción}

Como parte del programa de extensión y servicio ambulatorio del área de Clínica Médico-Quirúrgica de Grandes Animales de la Facultad de Medicina Veterinaria y Zootecnia de la Universidad de Córdoba, se atienden de forma gratuita, las necesidades clínicas de los equinos y bovinos de las diferentes explotaciones pecuarias del departamento de Córdoba, así mismo se instruye y orienta a los productores sobre las necesidades que los animales necesitan para que sean sanos y eficientes.

El conocimiento de la casuística clínica de los animales en determinada región, permite demostrar la importancia y el impacto que pueden estar causando las patologías en los diferentes sistemas de producción del Departamento, así como las posibles falencias, y quizás las estrategias de manejo y control que deben instaurarse como mecanismo preventivo. En este sentido, los estudios retrospectivos, son importantes ya que permiten la determinación de la frecuencia en que se producen las enfermedades, las características epidemiológicas y sus características clínico-patológicas (PIEREZAN et al., 2009).

Como ciencia, la epidemiologia estudia todos los factores envueltos en la ocurrencia de una enfermedad, su importancia está en reconocer la existencia de un problema, definir su causalidad, determinar su naturaleza y por tanto, ayudar a establecer un tratamiento que desenvuelva diferentes medidas de prevención, pudiendo ser de mucha importancia a la hora generar estrategias utilizando datos como la prevalencia y formas de presentación de las enfermedades (OLIVEIRA et al., 2010).

La medida más elemental de frecuencia de una enfermedad, o de cualquier otro evento en general, es el número de animales que la padecen o lo presentan (FREEMAN y HUTCHINSON, 1980) lo cual determina la importancia de un problema de salud determinado, pues debe referirse siempre al tamaño de la población de donde previenen los casos y el periodo de tiempo en el cual estos fueron identificados (CASTAÑEDA et al., 2005).

La práctica médica diaria requiere la toma de decisiones sobre actividades preventivas, diagnosticas, terapéuticas y pronosticas basadas en cálculo de probabilidades que pretenden delimitar la incertidumbre que envuelve el ejercicio profesional (BERWICH et al., 1981; ARGIMON y JIMÉNEZ, 2000). Estos aspectos se consideran más efectivos basados en estudios retrospectivos que arrojan resultados útiles para el futuro.
La importancia del diagnóstico correcto, resulta principalmente, en la realización de la estrategia terapéutica adecuada y exitosa, que debe comenzar con la historia, la evaluación del paciente y la escogencia de la prueba diagnóstica confiable (sensible y específica). La experiencia clínica del Médico Veterinario en el ejercicio profesional ayuda en la observación de elementos relacionados con la etiología de las enfermedades, ya que, según FEITOSA (2008), la mayoría de los errores médicos no se deben a un razonamiento defectuoso sobre hechos evaluados, ni al razonamiento bien conducido, pero si sobre hechos mal observados.

El conocer la frecuencia, la prevalencia o la incidencia de las enfermedades que más comúnmente presentan los pacientes que son atendidos en determinada región, permite poder planificar eficazmente la profilaxis adecuada, así como la adquisición de insumos, preparación de personal, acondicionamiento de instalaciones, adquisición de equipos, entre otras. En fin, esta información se convierte en una herramienta para la toma de decisiones en cuanto a la atención de las enfermedades. Por tal motivo, el presente trabajo tiene como objetivo analizar las consultas clínicas de los animales promovidos por el servicio ambulatorio del área de Clínica Médico-Quirúrgica de Grandes Animales, realizadas en el periodo comprendido entre 2008 y 2015 y determinar la frecuencia de presentación de las principales enfermedades que afectan a bovinos y équidos del Departamento de Córdoba.

\section{Materiales y Métodos}

El estudio fue realizado en fincas ubicadas en áreas rurales de diferentes municipios del departamento de Córdoba, el cual está ubicado entre las coordenadas $7^{\circ} 23^{\prime}$ y $9^{\circ} 26^{\prime}$ de latitud norte y los $74^{\circ} 52^{\prime}$ y $76^{\circ} 32^{\prime}$ de longitud oeste del meridiano de Greenwich, a una altura de $30 \mathrm{msnm}$, con temperatura promedio anual de $28^{\circ} \mathrm{C}$, humedad relativa del $82 \%$, precipitación media anual de $1400 \mathrm{~mm}$ y pertenece a la formación climática de bosque tropical lluvioso. Se presentan dos periodos bien definidos (época de lluvia y época seca) (PABÓN et al., 2001).

Fueron analizadas 3.308 historias clínicas a partir de los registros de los casos presentados entre los años 2008 y 2015 en el área de Clínica Médico-quirúrgica en Grandes Animales del servicio ambulatorio de la Facultad de Medicina Veterinaria y Zootecnia de la Universidad de Córdoba, las cuales cumplieron con los criterios de inclusión establecidos, siendo básicamente aquellas consideradas completas y que presentaban diagnóstico definitivo y que incluían la reseña, 
anamnesis, examen clínico general y específico, así como los medios auxiliares de diagnóstico necesarios como fueron hemograma, serología, raspado de piel, biopsias y coprológicos.

Todos los casos atendidos fueron clasificados considerando variables como: especie, sistema afectado, diagnóstico definitivo y tipo de tratamiento (Médico o Quirúrgico). La información fue almacenada y ejecutada en hojas de cálculo del programa Microsoft Excel, que sirvieron de base de datos para su posterior análisis descriptivo.

\section{Resultados y discusión}

La Tabla 1, muestra el total de historias clínicas analizadas (3.308), de las cuales 513 (14,5\%) correspondieron a casos clínicos en equinos y 2.795 $(85,5 \%)$ a casos clínicos en bovinos, de igual forma, se describe el número de casos según el año de presentación.

La marcada diferencia en la casuística clínica entre las dos especies se debe a que en el departamento de Córdoba predominan los sistemas de producción en bovinos, teniendo aún sistemas de tipo tradicional y semi-extensivo y muchas de las fincas donde fue prestado el servicio clínico ambulatorio, fueron de medianos y pequeños productores, donde la asistencia veterinaria no es de rutina, lo que podría aumentar la casuística en la especie bovina, así mismo, por ser explotaciones medianas o pequeñas, el inventario de los equinos estudiados era menor, por lo que fueron evaluadas las escasas poblaciones de equinos utilizados para trabajo en las diferentes producciones ganaderas.

En la Tabla 2, se relacionan los casos por sistemas afectados en los equinos y bovinos evaluados, por lo que se determinó que los casos con mayor frecuencia para ambas especies fueron aquellos que afectan al sistema sanguíneo y órganos hematopoyéticos, siendo la mayor frecuencia los hematozoarios con 79,8 (2.230) y $37,6 \%$ (193) para bovinos y equinos respectivamente. El sistema con la segunda casuística para ambas especies fue el de piel y anexos con 12,7 (355) y $26,12 \%$ (134) para bovinos y equinos respectivamente, siguen en su orden los sistemas digestivo, locomotor y musculo esquelético. Solo el 1,25\% (35) y el 5,65\% (29) correspondieron a procesos quirúrgicos en bovinos y equinos respectivamente.

La elevada casuística de hematozoarios (Babesia spp, Anaplasma spp) para ambas especies, pudo deberse a las condiciones agroecológicas propias del departamento de Córdoba que facilitan la mayor presentación de vectores transmisores como moscas, garrapatas y
Tabla 1. Casuística clínica en equinos y bovinos del servicio ambulatorio de la Clínica Médico-Quirúrgica de Grandes Animales en el periodo de 2008 al 2015.

\begin{tabular}{ccccc}
\hline & \multicolumn{4}{c}{ Casuística clínica ambulatoria } \\
\cline { 2 - 5 } Año & \multicolumn{2}{c}{ Equinos } & \multicolumn{3}{c}{ Bovinos } \\
\cline { 2 - 5 } & $\mathbf{N}^{\circ}$ & $\%$ & $\mathbf{N}^{\circ}$ & $\%$ \\
\hline 2008 & 112 & 21,8 & 453 & 16,2 \\
2009 & 105 & 20,5 & 397 & 14,2 \\
2010 & 45 & 84,9 & 432 & 15,5 \\
2011 & 128 & 25,0 & 949 & 34,0 \\
2012 & 17 & 3,3 & 135 & 4,8 \\
2013 & 22 & 4,3 & 98 & 3,5 \\
2014 & 31 & 6,0 & 76 & 2,7 \\
2015 & 53 & 10,3 & 255 & 9,1 \\
\hline Total & $\mathbf{5 1 3}$ & $\mathbf{1 0 0}$ & $\mathbf{2 . 7 9 5}$ & $\mathbf{1 0 0}$ \\
\hline
\end{tabular}

tábanos (CARDONA et al., 2012a). Reportes inferiores fueron realizados por CALDERON-GONÇALVES et al. (2011), quienes informaron frecuencias de $28,2 \%$ de hematozoarios en el estado de San Pablo, Brasil. Sin embargo, COSTAet al. (2011), reportaron frecuencias muy superiores que oscilaban entre el 2 y $80 \%$ de hematozoarios en el estado de Paraíba, Brasil. BUTLER et al. (2012) y NIJHOF et al. (2007), confirmaron transmisión autóctona de $B$. caballi y $T$. equi en equinos de zonas endémicas y concluyen que la presencia de Dermacentor reticulatus son las causas de los casos clínicos y de los anticuerpos detectados. CALDERÓN et al. (2013), reportaron prevalencia de Babesiosis en caballos de pesebreras del departamento de Córdoba muy por debajo con 18,25\%, atribuyéndoselo a que este tipo de caballos reciben manejo preventivo, sin embargo, SIGG et al. (2010) encontraron una mayor seroprevalencia en caballos importados desde zonas endémicas, ratificando la importancia del manejo preventivo y el control de vectores.

Son pocos los reportes de frecuencia, prevalencia, incidencia o casuística de enfermedades en bovinos del departamento de Córdoba, sin embargo, CARDONA et al. (2012b), reportaron una frecuencia de presentación de otitis parasitaria bovina en 6 explotaciones ganaderas de Córdoba del 63,2\%. De igual forma, fue reportada una frecuencia de Pythiosis cutánea bovina del $38,5 \%$ en 3 fincas ganaderas del departamento (CARDONA et al., 2012c; 2013a). Otras enfermedades descritas en bovinos del departamento de Córdoba son los carcinomas de células escamosas en bovinos mestizos Bos indicus $x$ Bos taurus despigmentados y sometidos a las altas temperaturas y exposición prolongada a rayos solares del trópico bajo (CARDONA et al., 2013b), así como dermopatías parasitarias como la demodecosis bovina (CARDONA et al., 2013c). 
Tabla 2. Casuística clínica según el sistema afectado en equinos y bovinos atendidos en el servicio clínico ambulatorio de la Universidad de Córdoba en el periodo de 2008 al 2015.

\begin{tabular}{|c|c|c|c|c|c|c|}
\hline \multirow{2}{*}{ Sistema Afectado } & \multicolumn{3}{|c|}{ Bovinos } & \multicolumn{3}{|l|}{ Equinos } \\
\hline & Diagnóstico & $\mathbf{N}^{\circ}$ & $\%$ & Diagnóstico & $\mathbf{N}^{\circ}$ & $\%$ \\
\hline $\begin{array}{l}\text { Sangre y órganos } \\
\text { hematopoyéticos }\end{array}$ & $\begin{array}{l}\text { Hematozoarios (Babesia spp, } \\
\text { Anaplasma spp). }\end{array}$ & 2230 & 79,78 & Hematozoarios (Babesia spp). & 193 & 37,62 \\
\hline \multirow{7}{*}{ Digestivo } & Enteritis catarral & 13 & 0,47 & Cólico por Impacción & 3 & 0,58 \\
\hline & Parasitismo & 47 & 1,68 & $\begin{array}{l}\text { Cólico por desplazamiento del colon } \\
\text { mayor }\end{array}$ & 1 & 0,20 \\
\hline & Coccidiosis & 19 & 0,67 & Enteritis Anterior & 2 & 0,40 \\
\hline & Neumoenteritis & 9 & 0,32 & Timpanismo Cecal & 1 & 0,20 \\
\hline & Salmonellosis, & 7 & 0,25 & Parasitismo & 16 & 3,12 \\
\hline & Sobrecarga ruminal. & 2 & 0,07 & Alteraciones Dentarias & 97 & 18,73 \\
\hline & Timpanismo ruminal & 11 & 0,40 & Colitis & 2 & 0,40 \\
\hline \multirow{6}{*}{ Piel y anexos } & Sarna Demodexica & 17 & 0,61 & Pythiosis cutánea & 58 & 11,31 \\
\hline & Dermatitis toxico alimentaria & 42 & 1,50 & Sarcoide fibroblástico & 24 & 4,68 \\
\hline & $\begin{array}{l}\text { Otitis parasitaria bovina (Rhabditis } \\
\text { spp) }\end{array}$ & 198 & 7,08 & Melanoma cutáneo & 15 & 2,92 \\
\hline & Papilomatosis & 38 & 1,40 & Habronemosis cutánea & 16 & 3,12 \\
\hline & Dermatofitosis (tiña). & 43 & 1,53 & Dermatofitosis & 18 & 3,51 \\
\hline & Carcinoma de células escamosas & 17 & 0,60 & Gurma bastara o metastásica & 3 & 0,58 \\
\hline \multirow{5}{*}{ Musculo esquelético } & Fractura de tibia & 1 & 0,04 & Arpeo idiopático & 3 & 0,58 \\
\hline & Ruptura de gastrocnemio & 1 & 0,04 & Enfermedad navicular & 5 & 0,97 \\
\hline & Necrobacilosis interdigital & 9 & 0,32 & Laminitis equina & 4 & 0,78 \\
\hline & Luxación de 3ra falange & 1 & 0,04 & Tendinitis flexora & 1 & 0,20 \\
\hline & Pezuña en tirabuzón & 15 & 0,54 & Bursitis bicipital & 1 & 0,20 \\
\hline \multirow{4}{*}{ Reproductor } & Retención de placenta & 2 & 0,08 & Balanitis ponzoñosa & 1 & 0,20 \\
\hline & Osqueítis ponzoñosa & 1 & 0,04 & Fractura de pene & 1 & 0,20 \\
\hline & Hidrocele & 2 & 0,08 & Hematocele & 2 & 0,40 \\
\hline & Acrobustitis & 11 & 0,40 & Metritis & 3 & 0,58 \\
\hline \multirow{6}{*}{ Cirugias } & Amputación de pezuña & 1 & 0,04 & Castración & 16 & 3,12 \\
\hline & Ruminotomía & 8 & 0,29 & Herniorafia umbilical & 1 & 0,20 \\
\hline & Descorne estético & 11 & 0,40 & Palatectomía (haba) & 6 & 1,17 \\
\hline & Desviación lateral del pene & 3 & 0,10 & Extracción de primer molar & 2 & 0,40 \\
\hline & Desmotomía apical dorsal & 4 & 0,14 & $\begin{array}{l}\text { Miotenectomía del extensor digital } \\
\text { lateral }\end{array}$ & 3 & 0,60 \\
\hline & Herniorafia umbilical & 7 & 0,25 & Enucleación & 1 & 0,20 \\
\hline \multirow[t]{3}{*}{ Respiratorio } & Bronquitis verminosa & 5 & 0,18 & $\begin{array}{c}\text { Obstrucción Recurrente de las Vías } \\
\text { Aéreas }\end{array}$ & 2 & 0,40 \\
\hline & Neumonía & 3 & 0,11 & $\begin{array}{l}\text { Hemorragia Pulmonar Inducida por } \\
\text { el Ejercicio }\end{array}$ & 1 & 0,20 \\
\hline & Edema pulmonar & 1 & 0,04 & Bronconeumonía & 3 & 0,60 \\
\hline \multirow[t]{2}{*}{ Metabólicas y carenciales } & Síndrome de la Vaca Caída & 2 & 0,08 & Rabdomiolisis & 1 & 0,20 \\
\hline & $\begin{array}{l}\text { Enfermedad del músculo blanco } \\
\text { (deficiencia de selenio) }\end{array}$ & 2 & 0,08 & $\begin{array}{c}\text { Esteatosis } \\
\text { (Deficiencia de selenio) }\end{array}$ & 1 & 0,20 \\
\hline \multirow{2}{*}{ Glándula Mamaria } & Mastitis clínica & 2 & 0,08 & \multirow{2}{*}{ Mastitis abscedativa } & \multirow{2}{*}{1} & \multirow{2}{*}{0,20} \\
\hline & Mamilitis Herpética Bovina. & 3 & 0,11 & & & \\
\hline \multirow{4}{*}{ Ojos y anexos } & Úlcera corneal & 2 & 0,08 & Úlcera corneal & 1 & 0,20 \\
\hline & Hipopión & 1 & 0,04 & Queratitis superficial & 2 & 0,40 \\
\hline & Queratitis pamnosa & 2 & 0,08 & $\begin{array}{l}\text { Obstrucción de conducto } \\
\text { nasolagrimal }\end{array}$ & 1 & 0,20 \\
\hline & Panoftalmia & 2 & 0,08 & Conjuntivitis traumática & 1 & 0,20 \\
\hline \multicolumn{2}{|c|}{ Total } & 2.795 & 100 & TOTAL & 513 & 100 \\
\hline
\end{tabular}


En su orden, las alteraciones dermatológicas, los problemas digestivos, los procedimientos quirúrgicos y las alteraciones locomotoras con $26,1 \%, 23,7 \%, 5,6 \%$ y $2,9 \%$ respectivamente, fueron destacados en la especie equina. Resultados inferiores fueron informados por De ALUJA et al. (2000), con 1,16\% 1,17\%, 3,58\% y $7,57 \%$, para las afecciones quirúrgicas, afecciones del sistema digestivo, afecciones locomotoras y dermatológicas respectivamente. PIEREZAN et al. (2009), reportaron frecuencias similares en afecciones del aparato digestivo, músculo-esquelético y tegumentar con $23,6 \%, 14,0 \%$ y $9,3 \%$ respectivamente.

Se han reportado frecuencias similares de enfermedades dermatológicas en equinos, es así como en el pantanal matogrossense de Brasil, fue reportada una frecuencia de pythiosis cutánea equina del $12,5 \%$ (SANTOS et al., 2011). Frecuencias superiores de dermopatías en equinos fueron informadas por SOUSA et al. (2011), quienes reportaron $57,4 \%$ de sarcoide y $8,3 \%$ de pythiosis del total de los casos en Santa María, Brasil. Mientras que CARVALHO et al. (2014), obtuvieron un $43,5 \%$ de sarcoide equino en caballos del Nordeste Brasilero. De igual forma, en un estudio retrospectivo realizado en Rio Grande do Sul, Brasil, el 23\% de las causas de muerte en equinos correspondió a neoplasias cutáneas y solo el $4,2 \%$ a pythiosis cutánea (MARCOLONGO-PEREIRA et al., 2014).

En Colombia, fue reportado en las sabanas de Bogotá, micosis y lesiones neoplásicas en el $20 \%$ de los casos (CASTELLANOS et al., 2014). De igual forma, en el departamento de Córdoba, CARDONA et al. (2014) reportaron una frecuencia de pythiosis cutánea equina del $26,9 \%$ en caballos de vaquería de 7 fincas productoras. Así mismo, se ha reportado la presencia de sarcoide equino de tipo fibroblástico, diagnosticado por métodos histopatológicos en Asnos (Equus asinus) del departamento (CARDONA et al., 2013d).

En el presente trabajo se evidenció los diferentes tipos y frecuencias de las patologías que afectan a los grandes animales del departamento de Córdoba, por lo que debe considerarse implementar estrategias para controlar las principales enfermedades que aquejan a estas especies, como por ejemplo, el control de vectores, el manejo adecuado de los de los neonatos y la detección temprana de los problemas dermatológicos como estrategia principal.

El médico veterinario se desempeña por lo general en el campo de la prestación de servicios de salud animal y en la mayoría de los casos, el éxito de su práctica profesional desde el punto de vista empresarial, se basa en la satisfacción y la percepción de calidad de sus clientes, por lo que conocer el estado epidemiológico de las enfermedades de los animales de su entorno, ayuda de alguna manera a tomar decisiones de tipo preventivo (ENGEL, 1976).

Agradecimientos: Al área de medicina y clínica de grandes animales de la Facultad de Medicina Veterinaria y Zootecnia de la Universidad de Córdoba.

\section{Referencias}

ARGIMON, P.; JIMÉNEZ, V. 2000. Métodos de investigación clínica y Epidemiológica. $2^{\circ}$ Ed. Harcourt. Madrid, España.

BERWICH, D.; FINEBERG, H.; WEINSTEIN, M. 1981. When doctors meet numbers. Am J med 71:991-998.

BUTLER, C.; SLOET VAN OLDRUITENBORGH, O.; STOUT, T.; VAN DER KOLK, J.; WOLLENBERG, L.; NIELEN, M.; JONGEJAN, F.; WERNERS, A.; HOUWERS, D. 2012. Prevalence of the causative agents of equine piroplasmosis in the South West of The Netherlands and the identification of two autochthonous clinical Theileria equi infections. Vet. J. 193 (2):381-385.

CALDERON GONÇALVES, R.; AMARAL DA SILVA, A.; LAURENTI FERREIRA, D.; BIAGIO CHIACCHIO, S.; SOUZA LOPES, R.; SECORUN BORGES, A.; MARTINS AMORIM, R. 2011. Tristeza parasitária em bovinos na região de Botucatu - SP: estudo retrospectivo de 1986-2007. Ciências Agrárias 32 (1):307-312.

CALDERÓN, A.; CARDONA, J.; VERGARA, O. 2013. Frecuencia de Babesia spp. en caballos de Montería, Córdoba (Colombia). Rev. U.D.C.A. Act. $\varepsilon$ Div. Cient 16 (2):451-458.

CARDONA, J.; ENSUNCHO, C.; VERGARA, O. 2012a. Frecuencia de hematrópicos en tres explotaciones de búfalos (Bubalus bubalis) del departamento de Córdoba, Colombia. Revista Científica, FCV-LUZ XXII (6):530-536.

CARDONA, J.; GONZÁLEZ, M.; ÁLVAREZ, J. 2012b. Frequency of clinical parasitic otitis due to Rhabditiform nematodes (Rhabditis sp) in six Gyr breed cattle farms in Cordoba, Colombia. Rev Colomb Cienc Pecu 25:417-421. 
CARDONA, J.; VARGAS, M.; PERDOMO, S. 2012c. Frequency of presentation of bovine cutaneous pythiosis (Pythium insidiosum) in three cattle farms in Cordoba, Colombia. Rev CES Med Vet Zootec 7 (2):47-54.

CARDONA, J.; VARGAS, M.; GONZÁLEZ, M. 2013a. Evaluación clínica e histopatológica de la Pythiosis cutánea en terneros del departamento de Córdoba, Colombia. Rev.MVZ Córdoba 18 (2):3551-3558.

CARDONA, J.; VARGAS, M.; PERDOMO, S. 2013b. Estudio Clínico e Histopatológico del Carcinoma de Células Escamosas de Bovinos en el Departamento de Córdoba, Colombia. Rev. Fac. Cs. Vets. UCV 54 (2):68-77.

CARDONA, J.; VARGAS, M.; PERDOMO, S. 2013c. Descripción clínica de la demodicosis bovina (Demodex bovis) en Córdoba, Colombia. Rev Inv Vet Perú 24 (1):125-129.

CARDONA, J.; VARGAS, M.; PERDOMO, S. 2013d. Estudio clínico e histopatológico del sarcoide fibroblástico en burros (Equus asinus) en Colombia. Revista Científica, FCV-LUZ XXIII (2):97-104.

CARDONA, J.; VARGAS-VILORIA, M.; PERDOMO, S. 2014. Frecuencia de pythiosis cutánea en caballos de producción en explotaciones ganaderas de Córdoba, Colombia. Rev. Med. Vet. Zoot 61 (I):31-43.

CARVALHO, F.; DANTAS, A.; RIET-CORREA, F.; ANDRADE, R.; NETO, P.; NETO, E.; SIMÕES, S.; AZEVEDO, S. 2014. Estudo retrospectivo das neoplasias em ruminantes e equídeos no semiárido do Nordeste Brasileiro. Pesq. Vet. Bras 34 (3):211-216.

CASTAÑEDA, L.; CALLES, M.; MORALES, R.; SALDIVIA, J.; RUIZ, L.; ORELLANA, N.; SANTELIZ, S.; DE LEON L.; BASTIDAS, Z.; GARCIA, M. 2005. Caracterización de la casuística atendida en el Hospital Veterinario "Dr. Humberto Ramirez Daza" del Decanato de Ciencias Veterinarias de la UCLA. Barquisimeto, Venezuela. Rev. Gaceta Cienc. Vet. $10(2): 119-122$.

CASTELLANOS, I.; PRADA, G.; RODRÍGUEZ, G.; SANTOS, R. 2014. Estudio microscópico de dermatopatías en equinos de la sabana de Bogotá, Colombia. Rev. Med. Vet 27:11-20.

COSTA, V.; RODRIGUES, A.; MEDEIROS, J.; LABRUNA, M.; SIMÕES, S.; RIET- CORREA, F. 2011. Tristeza parasitária bovina no Sertão da Paraíba. Pesq. Vet. Bras 31 (3):239-243.

DE ALUJA, A.; LÓPEZ, C.; CHAVIRA, S.; OSEGUERA, M. 2000. Condiciones patológicas más frecuentes en los équidos de trabajo en el campo mexicano. Vet. Méx 31 (2):165-168.

ENGEL, G. 1976. Are medical school neglecting clinical skills? J. Am. Med. Ass 36 (4):861-869.

FEITOSA, F. 2008. Semiología veterinaria - A arte do diagnóstico. Roca. São Paulo, Brasil.

FREEMAN, J., HUTCHINSON, G. 1980. Prevalence, incidence and duration. Am J epidermiol 112:707-723.

MARCOLONGO-PEREIRA, C.; ESTIMA-SILVA, P.; SOARES, M.; SALLIS, E.; GRECCO, F.; RAFFI, M.; FERNANDES, C.; SCHILD, A. 2014. Doenças de equinos na região Sul do Rio Grande do Sul. Pesq. Vet. Bras 34 (3):205-210.

NIJHOF, A.; BODAAN, C.; POSTIGO, M.; NIEUWENHUIJS, H.; OPSTEEGH, M.; FRANSSEN, L.; JEBBINK, F.; JONGEJAN, F. 2007. Ticks and associated pathogens collected from domestic animals in The Netherlands. VectorBorne and Zoonotic Diseases 7 (4):585-596.

OLIVEIRA, C.; OLIVEIRA, C.; HOLANDA, L.; BARBOSA, M.; ALBUQUERQUE, M.; MIRANDA, P.; SILVERIO, L.; FILHO, A.; FREITAS, A. 2010. Frequência epidemiológica das doenças infecto-contagiosas em cães atendidos no hospital veterinário da ufrpe. Disponíble en: http://www.sigeventos.com.br/jepex/inscricao/resumos/0001/R1171-1. PDF (Accesado 04/02/2016).

PABÓN, J.; ESLAVA, J.; GÓMEZ, R. 2001. Generalidades de la distribución espacial y temporal de la temperatura del aire y de la precipitación en Colombia. Meteorol. Colomb 4:47-59. 
PERUSIA, R. 2001. Casuística clínica regional en rodeos lecheros. Rev Inv Vet Perú 12 (2):123-134.

PIEREZAN, F.; RISSI D.; RECH, R.; FIGHERA, R.; BRUM, J.; BARROS, C. 2009. Achados de necropsia relacionados com a morte de 335 equinos: 1968-2007. Pesq. Vet. Bras 29 (3):275-280.

SANTOS, C.; SANTURIO, J.; MARQUES, L. 2011. Pitiose em animais de produção no Pantanal Matogrossense. Pesq. Vet. Bras 31 (12):1083-1089.

SIGG, L.; GERBER, V.; GOTTSTEIN, B.; DOHERR, M.; FREY, C. 2010. Seroprevalence of Babesia caballi and Theileria equi in the Swiss horse population. Parasitol. Int 59 (3):313-317.

SOUZA, T.; BRUM, J.; FIGHERA, R.; BRASS, K.; BARROS, C. 2011. Prevalência dos tumores cutâneos de equinos diagnosticados no Laboratório de Patologia Veterinária da Universidade Federal de Santa Maria, Rio Grande do Sul. Pesq. Vet. Bras 31 (5):379-382. 